\title{
New devices for diagnosis of atrial fibrillation: is necessary to treat with oral anticoagulation all patients?
}

\section{Editorial}

The technical possibility to diagnosis of Atrial Fibrillation is progressive ameliorate, and the number of asymptomatic patients affected by Atrial Fibrillation is increasing. For long time the ECG performed in patient with palpitation or stroke was the unique possibility to obtain a diagnosis of atrial fibrillation. For this reason a secondary Stroke was frequently in previously asymptomatic patients. A lot of patients that we consider "Asymptomatic patients" were been recognized only by cardiac Holler. In this exam unfrequently were present short episodes of atrial fibrillation.

More recently, an incremental using of Pacemaker, Loop Recorder and ICD for therapy permits to reveal some cases of asymptomatic atrial fibrillation. Recent technology developments indeed point towards wearables for example smart watches, this especially being quite effective and representing a novel method for screening for silent FA in the general population. Using these to perform cardiac monitoring, the possibility to diagnosis the atrial fibrillation was increased to $84 \%$.

We live in a time where the number of Asymptomatic Patients is very increased, a lot of these Patients have comorbidities, elderly age and risk for thromboembolic events. For several studies the Asymptomatic Patients have a minor risk for mortality than symptomatic patients. The recent guidelines of EHRA society shows that in a community of patients with atrial fibrillation, reported a risk of stroke reduced in asymptomatic patients less than $15 \%{ }^{1}$

Different Country started screenings of population for detect atrial fibrillation and Heart Anomalies. Is justify to treat with oral anticoagulation the asymptomatic patients?

A recent Swedish Study based on screening population, said that $87 \%$ still Asymptomatic for Atrial Fibrillation. When found FA started fast the anticoagulation treatment, so is made a futures prospective area (5 years). In this time the patient have a reduction Risk for Stroke and a reduction of possibility to have events that can lead to death. ${ }^{2}$

Depend on recent guidelines the treatment of atrial fibrillation based on CHAD-VASC risk score and longtime of episodes.

In Finland a several years ago with a screening Study found a correlation about dimension Atrium Cardiac and Atrial fibrillation. More is large the Cardiac Atria and more is possible that in a short time the patients have a massive event that leads to death. The CHAD-Vasc score is in depended of heart disease. The Cardiologist didn't' $t$ make difference between a patients with atrial fibrillation with symptoms and asymptomatic, with heart disease or without heart disease, etc., So two different typologies patients are involved in a single common Therapeutics area.

Probably could be useful in asymptomatic patient evaluate the heart structure also. We don't know the futures and the cardiology doesn't know the develop therapeutic area of Asymptomatic patients. Asymptomatic patients treated with oral anticoagulation must be
Volume 14 Issue 4 - 202 I

\author{
Giovanni Fazio, Randazzo Natale Eliseo \\ 'Department of cardiology, internal medicine, angiology and long \\ term care - Triolo Zancla Hospital -Palermo, Italy
}

Correspondence: Dr. Giovanni Fazio, Department of cardiology, internal medicine, angiology and long term care Triolo Zancla Hospital -Palermo, Italy, Tel +393334439962, Email Faziogiova@gmail.com

Received: June 30, 202I | Published: July 06, 2021

analyzed more, routine Echocardiography or others examinations cold be the key to revel a patient that go away to stroke.

Today for the question: "With the new devices for diagnosis of atrial fibrillation, is necessary to treat with oral anticoagulation all patients?" there is not a real certain answer but with the future we will have indications and studies so as to have a certain answer.

\section{Acknowledgments}

None.

\section{Conflicts of interest}

Author declares there are no conflicts of interest.

\section{Funding}

This manuscript was not supported by any funding.

\section{References}

1. Zhang J, Lenarczyk R, Marin F et al. The interpretation of CHA2DS2VASc score components in clinical practice: a joint survey by the European Heart Rhythm Association (EHRA) Scientific Initiatives Committee, the EHRA Young Electrophysiologists, the Association of Cardiovascular Nursing and Allied Professionals, and the European Society of Cardiology Council on Stroke. Europace. 2021;23(2):314-322.

2. Kostantinos C, Bernard J, Pamela McCabe, et al. "Typical Atypical and Asymptomatic presentation of new -onset atrial fibrillation in the community: Characteristics and prognostic implications. Heart Rhythm. 2016;13(7):1418-1424.

3. Engdahl J, Holmen A, Rosenqvist M, et al. A prospective 5- year's followup after population -based systematic screening for atrial fibrillation. Europace. 2018;20(FI_1):306-311. 